\title{
Evaluation of the Rationality of Urban Park Spatial Layout Based on Disaster Prevention Objective
}

\section{-Taking Yuhang District of Hangzhou as an Example}

\author{
Ju Zeng, Wenjun Zhang, Jiaxuan Chen, Xin Cheng \\ School of Civil Engineering and Architecture, Southwest University of Science and Technology, Mianyang, China \\ Email: 1593327368@qq.com
}

How to cite this paper: Zeng, J., Zhang, W.J., Chen, J.X. and Cheng, X. (2022) Evaluation of the Rationality of Urban Park Spatial Layout Based on Disaster Prevention Objective. Open Access Library Journal, 9: e8496.

https://doi.org/10.4236/oalib.1108496

Received: February 16, 2022

Accepted: March 6, 2022

Published: March 9, 2022

Copyright $\odot 2022$ by author(s) and Open Access Library Inc.

This work is licensed under the Creative Commons Attribution International License (CC BY 4.0).

http://creativecommons.org/licenses/by/4.0/

(c) (i) Open Access

\begin{abstract}
The disaster prevention function of urban parks plays a role in disaster prevention, refuge, rescue and reconstruction when the city is faced with disasters. Taking 32 urban parks with the function of disaster prevention and hedging in Yuhang District as the research object, the spatial layout of urban parks in the research area was analyzed by using the road traffic network accessibility and facility service area analysis tool of ArcGIS. The results showed that the distribution of urban parks in Yuhang district was concentrated in the east and south, and the number of urban parks in the northwest was small. Under the influence of traffic accessibility, the service domain of urban parks in northwest China is obviously smaller than that in eastern and southern China. Even within the service radius of urban parks, affected by the accessibility of traffic, there will be the situation that urban parks cannot be effectively covered. In view of the unreasonable layout of urban parks in Yuhang district, the following suggestions are put forward: the spatial layout of disaster prevention parks should be balanced layout considering accessibility factors; the space layout pattern of park green space nested with each other; Establish linkage mechanism of urban green space.
\end{abstract}

\section{Subject Areas \\ Urban Planning}

\section{Keywords}

City Park, Service Area, Accessibility, Spatial Layout 


\section{Introduction}

In the era of ecological civilization, sponge city, resilient city, park city and healthy city have been proposed to solve the increasingly prominent urban problems, aiming to build a more livable, safer and healthier urban space. In view of this, the spatial layout planning of urban parks based on the goal of disaster prevention and hedging has become the core issue of urban planning Urban park is the green infrastructure of a city. As the main public open space of a city, urban park generally has a variety of value systems, and undertakes multiple functions to maintain the sustainable development of the city. It plays a great role in the protection of urban fire prevention, disaster prevention and risk aversion.

Developed countries attach great importance to the construction of disaster prevention parks. In 1923, Japan experienced the Great Ganzhong earthquake. Post-earthquake research found that after the earthquake, the number of people who took refuge in the three parks of Ueno Park, Shiba Park and Fukagawa Sumi accounted for half of the total number of people who took refuge in Tokyo, and $70 \%$ of the population of Tokyo at that time, namely 1.57 million citizens, took the park as a refuge [1]. Since then, Japan has made corresponding studies on the layout of urban disaster prevention parks and the design of disaster prevention and risk aversion functions, and the planning and construction of disaster prevention parks are quite mature. Japan first proposed the concept of "disaster prevention parks" [2].

China's urban parks have long adopted the planning strategy of "building first and green space filling in the space" [3], which makes the park green space fragmented and uneven in space and makes it difficult to form a complete and balanced urban green space system, seriously affecting the equality and effectiveness of social benefits. With the rapid development of urbanization in our country, urban environmental problems are surging accordingly, which means that the planning concept of urban parks should keep pace with The Times. Urbanization development in our country is in the high speed of urbanization to high-quality urbanization is the important period of transition, on the one hand, the population of urban agglomeration is becoming more and more building density is increasing at a rapid pace, on the other hand, the problems in the urban development of urban governance also gradually revealed, especially from the new champions league since the outbreak, people pay more attention to the city in the face of sudden disaster self-protection ability, Are also increasingly aware of the vulnerability of and uncertain factors are on the increase of social development, in the event of disasters, especially in fast speed, wide range of influence, the destructive disasters, such as earthquake, fire, how to quickly and efficiently to the affected people in the shortest possible time to evacuate safely transfer to a safe refuge, the biggest problem is the urban development [4]. Therefore, reasonable division of asylum service domain is a very important premise to ensure the safety of asylum population. 
In recent years, urban managers and many scholars have attached great importance to the evaluation of spatial layout rationality of urban parks. In 2018, the Ministry of Housing and Urban-Rural Development issued guidelines for Disaster Prevention and Hedge Design of Urban Green Space, proposing that the service radius of urban green space for disaster prevention and hedge function should be set according to the disaster prevention and hedge planning of urban green space system. Li Jingqi et al. [5] studied the planning principles and layout of disaster prevention parks, and preliminarily discussed the urban disaster prevention park system and refuge space planning. Zhang Canqiang et al. [6] took the main urban area of Beijing as an example to set the service radius of different types of green space with hedging function and divide the scope of its service domain. Fu Sunping et al. [7] took Licheng District and Fengze District of Quanzhou City as an example, took the disaster prevention park in the area as one area, set the service radius of each disaster prevention park, and delineated the service domain of disaster prevention park. Taking Huangpu District of Shanghai as an example, Ye Mingwu et al. [8] used the network analysis model to analyze the service domain of the park by referring to the service radius standard stipulated for refuge places. Li Xiaoling et al. [4] took Changchun city as an example to analyze the spatial structure of urban parks with neighborhood method and evaluate the effectiveness of park refuge. However, based on the purpose of disaster prevention in urban parks, the service scope of disaster prevention park is determined according to the subjective service radius of disaster prevention park, which is obviously inconsistent with reality. The traffic network is the primary factor affecting whether the disaster prevention site can be reached quickly in the event of disasters. Based on the actual road network distance, this paper simulates that residents can reach the disaster prevention park space in the shortest time when disasters occur, and calculates the service scope of the park. Compared with the previous subjective method of demarcating service radius, it is obviously more accurate and scientific to calculate the service domain according to the actual road network distance. Through the visualization analysis of the service domain and accessibility of urban parks by using geographic information system (GIS), the defects and blind areas in the spatial layout of disaster prevention parks were identified, in order to provide a favorable basis for optimizing the spatial layout of disaster prevention parks.

\section{Research Area and Research Method}

\subsection{Overview of the Study Area}

Yuhang District, as the research object of this paper, is located in the east, west and north of Hangzhou, half surrounded by the central urban area of Hangzhou, with a total area of 1228.41 square kilometers and jurisdiction over 6 towns and 14 streets. By the end of 2019, the registered population of the district was 1.1618 million and the permanent resident population was 1.891 million. In 2019, The 
annual GDP of Yuhang District was 282.4 billion yuan, ranking first among all the districts and counties (cities) in Hangzhou. In the face of huge economic position and urban population pressure, in order to ensure residents enjoy a healthier, safer and livable living environment, the planning of urban parks in the region is very important. In August 2020, in order to effectively improve the level of park construction and management, speed up the building of "park city", and strive to "make parks a green space shared by the people", Hangzhou published the second batch of park lists. There are 32 in Yuhang District (Table 1), ranking first among all districts and counties (cities).

\subsection{Data Sources}

The attribute data of Yuhang District is mainly obtained through Google map and field survey. The specific location and data of urban parks are obtained through Google map and imported into ArcGIS to generate data layers of urban parks (Table 1). The road network data of Yuhang district were obtained by national Geographic Information Resource Directory Service system and OpenStreetMap (ESRI) in ArcGIS.

\subsection{Research Methods}

\subsubsection{Division and Processing of Urban Park Service Domain}

The size of park green space and the improvement of infrastructure will affect the service domain of its disaster prevention function. Small emergency disaster prevention park suitable for medium fixed disaster prevention park suitable for large central disaster prevention park. Japan specifically regulates the level of disaster prevention parks according to the area of disaster prevention parks. Parks with an area of more than $1 \mathrm{hm}^{2}$ are the first refuge places that people go to after the occurrence of disasters, and all parks should be built as transfer stations with the function of disaster prevention and risk aversion [1]. Based on the mature experience of Japan, this study divides the service radius of urban parks into three grades, $500 \mathrm{~m}$ is the geographical scope that can reach the disaster prevention park within 3 minutes after the disaster occurs. $1500 \mathrm{~m}$ is the area 10 minutes away from the park after the disaster. $2500 \mathrm{~m}$ is the reachable area $1 \mathrm{~h}$ after the disaster (Figure 1). This study divides the service radius based on the traffic distance of the urban road network rather than the dotted circle of the park.

\subsubsection{Visualization of Accessibility}

Transportation accessibility analysis is an important factor that must be considered in urban planning, and plays an important role in infrastructure layout, road network optimization, land price evaluation, location analysis, etc. [9]. The accessibility of urban parks can be analyzed based on the accessibility of urban traffic network, mainly studying the relationship between the spatial distribution of areas with good or insufficient accessibility and the location of urban parks. If the site selection of urban parks is positively correlated with the accessibility of 
Table 1. List of urban Parks in Yuhang district, Hangzhou.

\begin{tabular}{|c|c|c|c|c|}
\hline Number & Name & Own Street & Longitude & Latitude \\
\hline 1 & Linpingshan Park & Linping Street & 120.28455759 & 30.42042706 \\
\hline 2 & South Lake Park & Nanhu Street & 119.75792574 & 30.38661340 \\
\hline 3 & sports Park & Xingqiao Street & 120.25165482 & 30.39355844 \\
\hline 4 & Tiandu Park & Linping Street & 120.24444717 & 30.39636926 \\
\hline 5 & Mount Royal Park & Donghu Street & 120.28033898 & 30.42572104 \\
\hline 6 & Shuiting East Lake Park & Linping Street & 120.28775844 & 30.43456036 \\
\hline 7 & beautiful island park & Liangzhu Street & 120.02999690 & 30.38032797 \\
\hline 8 & Tower Hill Park & Yuhang Street & 119.94722161 & 30.27384658 \\
\hline 9 & $\begin{array}{l}\text { Liangzhu Culture } \\
\text { Village Mine Park }\end{array}$ & Liangzhu Street & 119.97299728 & 30.30255637 \\
\hline 10 & Pingyao Yaoshan Park & Pingyao Town & 119.96428035 & 30.39656503 \\
\hline 11 & water park & Linping Street & 120.29393288 & 30.40310732 \\
\hline 12 & $\begin{array}{l}\text { Liangzhu Cultural } \\
\text { Village Park }\end{array}$ & Liangzhu Street & 120.02352106 & 30.37087043 \\
\hline 13 & Fangjiashan Park & Xianlin Street & 119.98466675 & 30.21839814 \\
\hline 14 & century Park & Donghu Street & 120.30470761 & 30.44176669 \\
\hline 15 & People's Square Park & Nanyuan Street & 120.30924818 & 30.37489143 \\
\hline 16 & Longxing Park & Nanyuan Street & 120.30889728 & 30.40614617 \\
\hline 17 & $\begin{array}{l}\text { European and American } \\
\text { Financial City Park }\end{array}$ & Cangqian Street & 120.00596617 & 30.28305701 \\
\hline 18 & $\begin{array}{l}\text { Wujiamen Road } \\
\text { Landscape Park }\end{array}$ & Chongxian Street & 120.11775326 & 30.37958064 \\
\hline 19 & $\begin{array}{l}\text { Future Technology City } \\
\text { Central Park }\end{array}$ & Cangqian Street & 119.99874434 & 30.27605438 \\
\hline 20 & $\begin{array}{c}\text { Chongxian } \\
\text { Wolongbang Park }\end{array}$ & Chongxian Street & 120.16237981 & 30.37874754 \\
\hline 21 & Neon Road Landscape Park & Liangzhu Street & 120.12156211 & 30.38031887 \\
\hline 22 & Wushan Park & Jingshan Town & 120.16422781 & 30.23439724 \\
\hline 23 & $\begin{array}{l}\text { Chongxian } \\
\text { Four-dimensional Park }\end{array}$ & Chongxian Street & 120.17545847 & 30.38750818 \\
\hline 24 & Liangzhu Ecological Park & Liangzhu Street & 120.07038422 & 30.36291860 \\
\hline 25 & Changle Park & Jingshan Town & 119.86521691 & 30.37509508 \\
\hline 26 & Baolin Road Fertility Garden & Nanhu Street & 119.94351549 & 30.26779998 \\
\hline 27 & $\begin{array}{c}\text { Shishan Road Nanyan } \\
\text { Landscape Park }\end{array}$ & Yuhang Street & 119.94967029 & 30.26565517 \\
\hline
\end{tabular}




\begin{tabular}{ccccc} 
Continued & & & & \\
\hline 28 & Tangqi Park & Tangqi Town & 120.18277499 & 30.47559134 \\
29 & Huali Park & Wuchang Street & 119.87683022 & 30.25400865 \\
30 & South Star Pocket Park & Xingqiao Street & 120.25974722 & 30.39284423 \\
31 & Liangzhu Park & Liangzhu Street & 119.98834302 & 30.38676950 \\
32 & Gouzhuang Park & Liangzhu Street & 120.08673259 & 30.35391835 \\
\hline
\end{tabular}

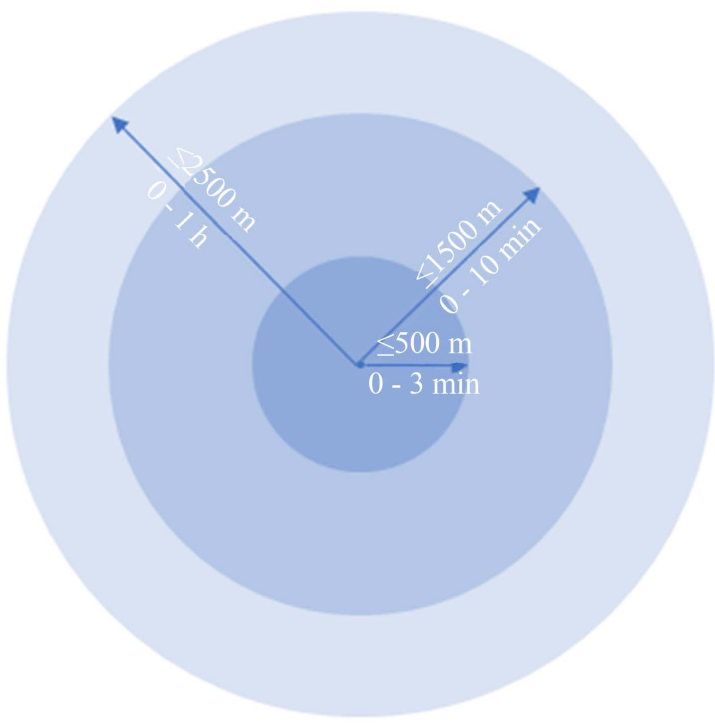

Figure 1. Service radius division of urban parks.

urban traffic, the layout of urban disaster prevention parks is more reasonable. When the city is faced with sudden natural disasters, people can reach the urban parks in a short time to prevent and avoid disasters. Otherwise, it indicates that the layout of urban parks needs to be optimized, and measures need to be taken to guide and strengthen urban parks and urban transportation networks. Based on the accessibility analysis of urban transportation network, this study analyzes the accessibility degree from each location in the region to any other location by using the accessibility evaluation method of minimum impedance. Basic steps: 1) Prepare administrative boundary layer and road network data of Yuhang District. 2) ArcGIS is used to construct the traffic network model of Yuhang District and perform assignment operation on the traffic network model. Enable [New OD Cost Matrix] tool under ArcGIS network analysis function to calculate the shortest travel time from each intersection to other intersections. 3) The accessibility of each intersection and road network was counted, and finally the map was visualized. The [spatial interpolation] analysis tool was used and the [inverse distance weight method] was used to generate the accessibility distribution map. The higher the accessibility value, the better the accessibility.

\subsubsection{Service Domain Analysis}

Based on the urban road traffic network model established by ArcGIS, the ser- 
vice domain is analyzed. Enable [New service area] tool in [Network Analyst] in ArcGIS, load facility point option into 32 urban parks in this study, set impedance factor as [distance (meter)], [default interruption] as [500 1500 2500], and then compute service domain. In order to more intuitively show the difference between the service domain research based on road network and the service domain research based on circle drawing with radius, this paper also makes a comparison with the operation based on circle drawing with radius drawing.

\section{Results and Analysis of Fruit Fractions}

\subsection{Accessibility of Urban Transportation Network}

The accessibility of transport network in the study area is obviously different, mainly reflected in that the accessibility of the eastern region is significantly higher than that of other regions. The eastern region belongs to Linping Street of Yuhang District, which is close to the main city of Hangzhou and has relatively complete infrastructure construction, dense road network and high regional accessibility. In the central region, Liangzhu Street, Cangqian Street and Xianlin Street have good accessibility (Figure 2).

\subsection{Accessibility of Urban Parks}

Based on the analysis of urban transport accessibility, 32 parks were embedded into the map of urban transport network accessibility. It can be seen that the spatial distribution of urban parks in Yuhang district is basically consistent with the accessibility of urban transport network, and they are mainly located in the

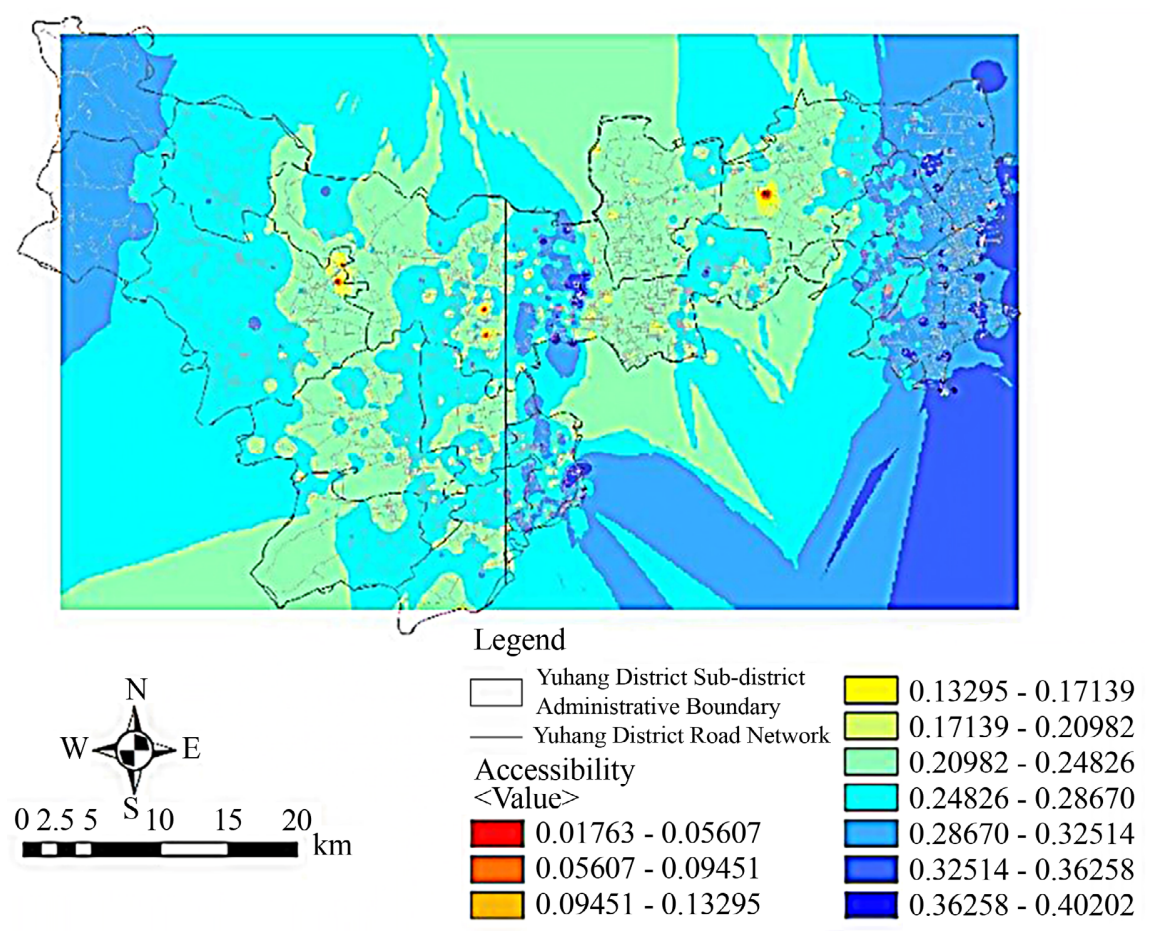

Figure 2. Accessibility of the traffic road network in Yuhang District. 
areas with high accessibility in the city. There are 9 urban parks concentrated in the eastern region, 7 urban parks distributed in the central region, and 8 urban parks distributed in the southwest of Yuhang District near the central city of Hangzhou. The accessibility of the western region is not high, and only two parks are located in this area (Figure 3).

\subsection{Analysis of Urban Park Service Domain}

In the construction of the urban traffic network model, on the basis of analysis of city park service domain (Figure 4), according to the calculation of set can be seen that the impedance of the radius of 32 city park $2500 \mathrm{~m}$ distance service domain range covers most of the study area, the eastern region scope of service domains of city parks was obviously higher than that of the western region. Because of this study is calculated according to the actual road network, will be even park distribution are all around but still cannot be effective coverage of city park situation, such as a central region was not completely covered city park in the accessibility of the urban traffic network diagram also showed relatively weak accessibility features. In order to illustrate the situation more directly and demonstrate the effectiveness of the research method, this study also adopts the method of establishing core buffer, draws a circle with the same path distance as the radius, and shows the method of drawing a circle with the service radius to find the service domain of urban park (Figure 5). The service domain drawn from the circle of the service radius of urban parks is obviously different from that obtained by the method adopted in this study. First of all, its service domain is a regular circle, which is inconsistent with the actual situation. If the park is

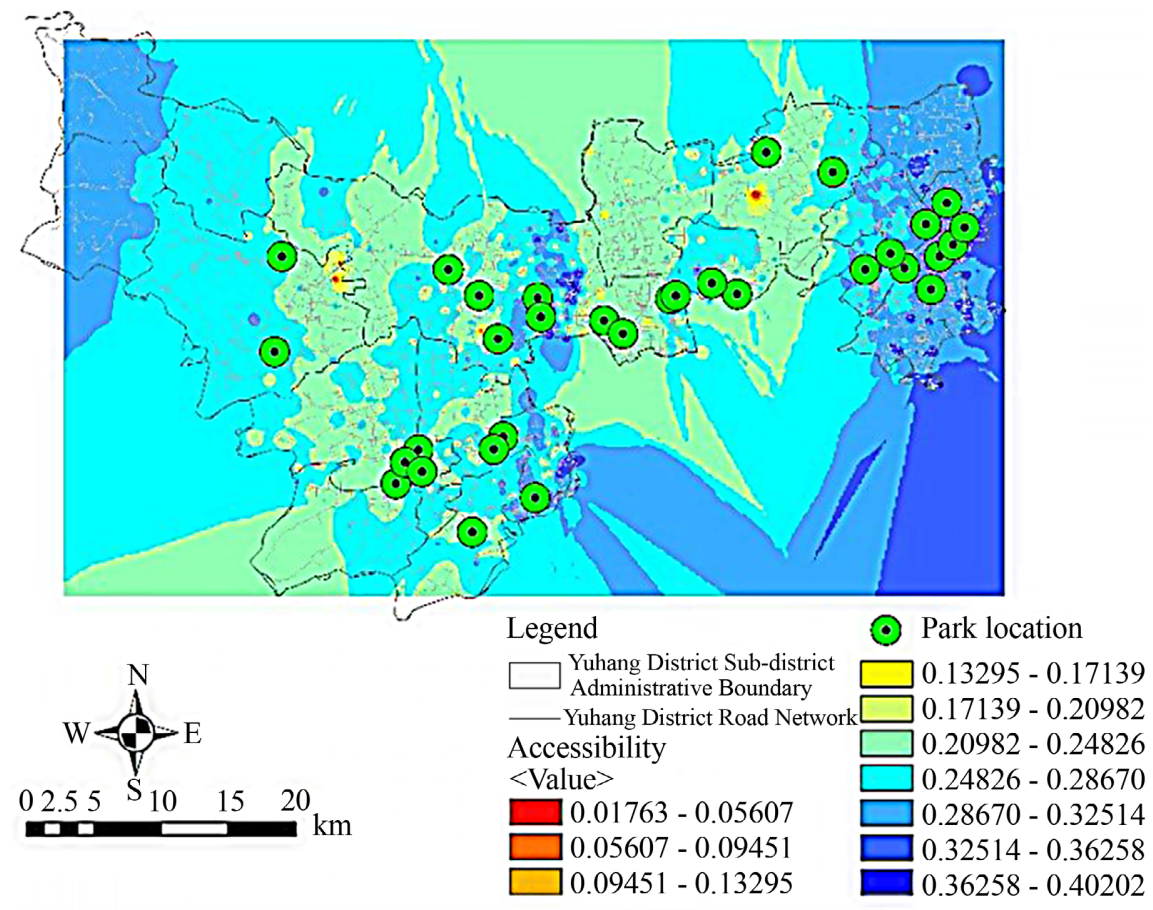

Figure 3. Analysis of accessibility of urban parks in Yuhang District. 


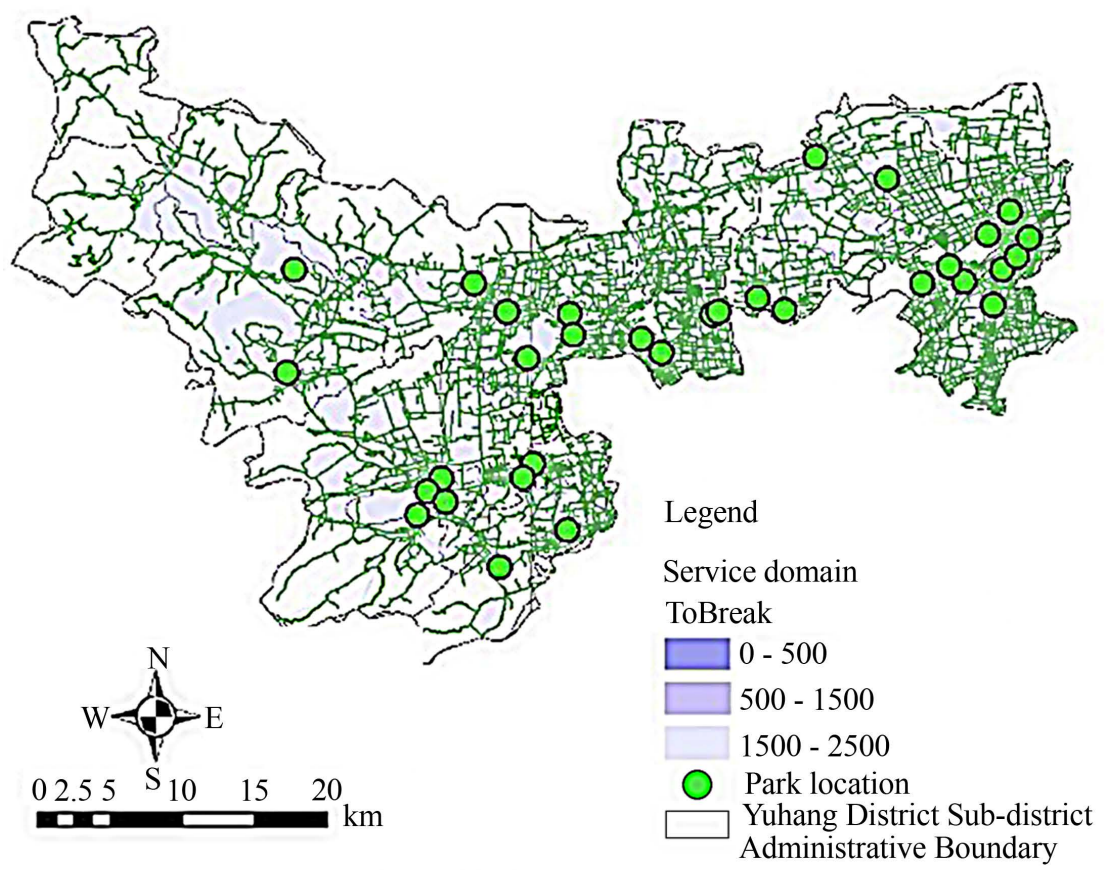

Figure 4. Service scope of urban park based on urban road network.

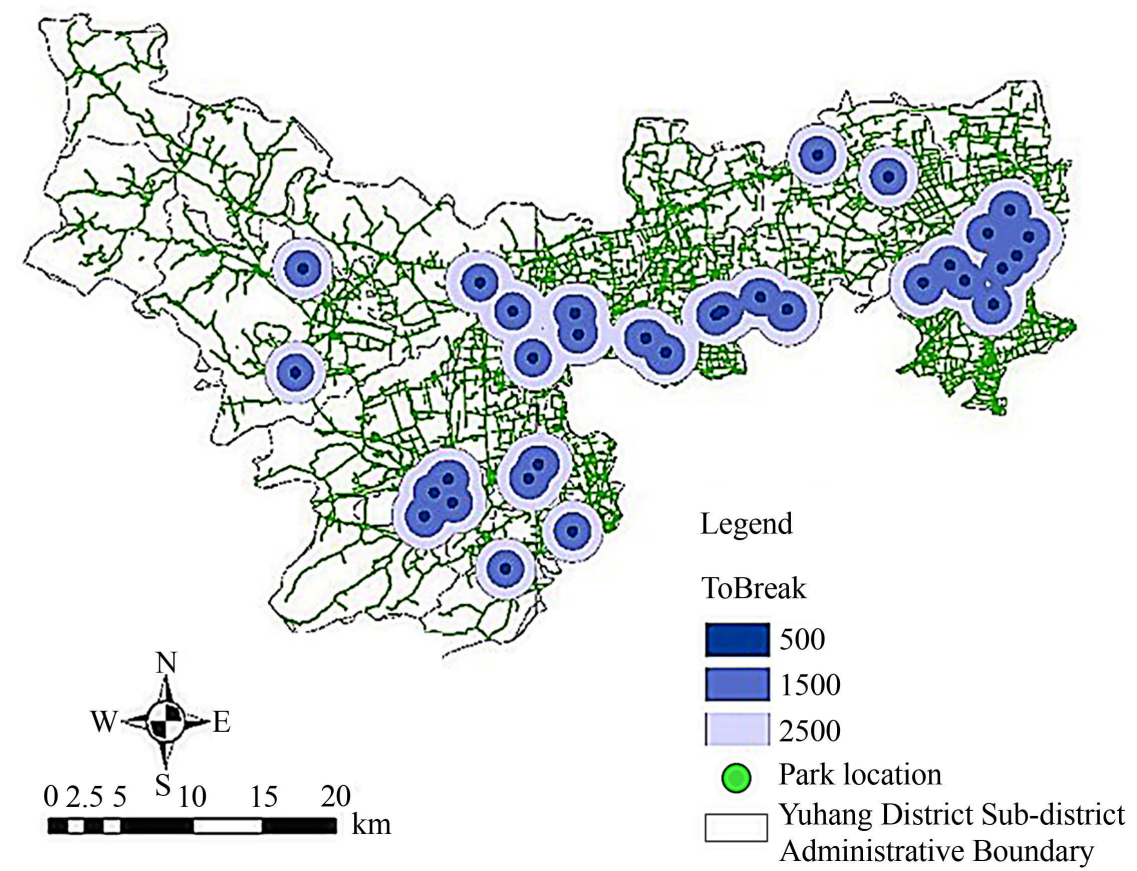

Figure 5. Urban park service domain based on core buffer.

located in an area with weak traffic accessibility, residents within the coverage range of $2500 \mathrm{~m}$ cannot enter the urban park in time to avoid disasters.

\section{Analysis of Results}

In this paper, ArcGIS is used to simulate residents' choice of urban refuge places under the principle of proximity when disasters occur, so as to divide the service 
domain of urban parks. Compared with the service domain division method drawn in a circle according to the service radius standard, the actual distance of urban road network and the accessibility factor of urban road network are adopted in this study to delimit the service domain, and the result is more consistent with the reality. Based on the division of urban park service domain, this study analyzes the spatial distribution of urban parks in Yuhang District, and draws the following conclusions:

1) The spatial distribution of urban parks is not balanced, the number of parks in the east and south is dense, and the number of parks in the northwest is sparse. The northwest is mostly hilly area with weak transportation accessibility. Urban parks serve as disaster shelter sites and relief materials distribution centers. When disasters occur, it will take a long time to reach the disaster shelter sites in the northwest and obtain relief materials for a long time, which is not conducive to post-disaster emergency evacuation and management.

2) The northwest area of urban park service area is significantly lower than other areas. Through the analysis of urban traffic accessibility and spatial distribution characteristics of urban parks, it can be seen that there are two main reasons for the weak radiation of urban parks in northwest China. One is that this region belongs to Baizhang Town and Corniniao town, and there are few traffic channels with the central region, and its traffic accessibility is obviously weaker than that of the eastern region. In addition, the northwest region belongs to dajingshan region, where the demand for the greening and recreation function of urban parks is obviously lower than that of other regions, so the number of parks in this region is also small.

3) Blind areas of service domain coverage exist even in densely populated areas of urban parks. In reality, the accessibility of a region is affected by multiple factors. Therefore, there may be areas near the city park that cannot reach the city park in time. In Jingshan town, bottle kiln town and yuhang street at the junction of such a situation.

\section{Conclusion and Discussion}

From the perspective of the accessibility of urban park service areas, this study uses ArcGIS software to analyze the spatial layout of urban parks in the study area, and provides a favorable basis for optimizing the spatial layout of disaster prevention parks.

The distribution of urban parks in Yuhang District is concentrated in the east and south, and the number in the northwest is small; under the influence of traffic accessibility, the service area of urban parks in the northwest is significantly smaller than that in the east and south; Traffic accessibility will also affect the situation that cannot be effectively covered by urban parks.

In view of the above deficiencies, based on the objective of urban disaster prevention, several suggestions are put forward for the planning of urban parks in Yuhang District: 1) The spatial layout and site selection of disaster prevention 
parks should consider accessibility factors and arrange them as balanced as possible. Considering the accessibility factors of the urban road network and the service radius of urban parks and other influencing factors, according to the service radius of parks and green spaces at all levels in the city and the accessibility of urban traffic locations, scientific layout, on the basis of green space system planning, build a park and green space system with a reasonable hierarchical structure enhances the carrying capacity of urban refuge. The future urban park planning site selection of Yuhang District should focus on the northwest area, and the number of urban parks can be appropriately increased. 2) The spatial layout pattern of park green space nested with each other. Urban disaster prevention green space is divided into different types and levels, which undertake different tasks in the process of urban disaster prevention, hedging, disaster relief and post-disaster reconstruction. This study found that there are still blind areas in the 32 urban park service areas studied, and urban parks alone cannot form a good coverage. So green space in urban disaster prevention planning but basis according to the urban traffic network service domains of the accessibility of city parks, park in the city cannot effectively cover set different levels of green space, this kind of Mosaic space layout mode to ensure the efficient and effective coverage of the urban green space in disaster prevention, to facilitate rapid dispersion of post-disaster relief material and post-disaster emergency management [4]. 3) Establish linkage mechanism of urban green space system [10]. Urban disaster prevention park is an important part of urban disaster prevention green space system. In the planning of improving urban resilience, urban disaster prevention park is not a single battle. All kinds of green space for disaster prevention and hedging should be connected with each other through transportation network and information network to establish linkage mechanism between each other. In order to achieve the optimal allocation of urban disaster prevention park in spatial layout and internal space organization in the process of resilient city construction, and the cross and integration of disaster prevention park and other green space organizations in the city can make the disaster prevention and hedge system in the urban green space system form a closed loop [11]. Urban emergency shelter is a complex space system, based on the goal of disaster prevention of urban park is just one of the important constituent, in addition to square, schools and other subsystems, this study only in urban park space layout is reasonable, for example, research on the accessibility of transportation network factors under the influence of urban park service domain, try the layout of the evaluation system of urban disaster prevention park, The next research direction is to include different levels of urban disaster prevention green space and other urban emergency refuge places on this basis, and evaluate the spatial layout of the entire urban emergency refuge system. Due to data limitations, the selection of urban parks in this study is not comprehensive. It is only based on the 32 parks in Yuhang District published by Hangzhou City, which may be overlooked. Second analysis in this study did not consider the factor of urban 
population, population scale is a measure of urban disaster prevention park radiation ability of disaster prevention safety an important indicator of park space layout and the matching of the size and population distribution is the important factors that affect the spatial layout, population factor needs to be addressed in future studies.

\section{Conflicts of Interest}

The authors declare no conflicts of interest.

\section{References}

[1] Wang, P. and Lu, X. (2020) Japan's Disaster Prevention Park System Construction and Experience Reference. China Disaster Reduction, No. 19, 56-59.

[2] Long, D., Zhang, X.C. and Dai, F. (2011) Discussion on the Research and Construction of Disaster Prevention Park in My Country under the New Situation. Modern Urban Studies, 26, Article No. 5762.

[3] Zhang, J.G. and Zhao, B. (2018) Study on Site Selection and Layout Optimization of Urban Parks Based on Accessibility. Journal of Nanjing Forestry University (Natural Science Edition), 42, 151-158.

[4] Li, X.L., Xiu, C.L., Cheng, L. and Wang, N.Y. (2016) Spatial Structure and Rationality Evaluation of Urban Parks Based on Disaster Prevention Objectives: Application of Neighborhood Method in Changchun City. Journal of Applied Ecology, 27, 3641-3648.

[5] Li, J.Q. and Xia, X. (2007) Research on Urban Disaster Prevention Park Planning. Chinese Garden, No. 7, 16-22.

[6] Zhang, C.Q., Zhang, B., Li, W.H., et al. (2012) Evaluation of the Function of Disaster Prevention and Avoidance of Green Space in Beijing Urban Area. Geographical Research, 31, 2301-2309.

[7] Fu, S.P. and Liang, C.L. (2011) Research on the Spatial Layout of Urban Disaster Prevention Park Based on Service Domain-Taking Quanzhou City as an Example. City and Disaster Reduction, No. S1, 56-59.

[8] Ye, M.W., Wang, J., Chen, Z.L., et al. (2009) Comprehensive Decision-Making Analysis of Urban Disaster Prevention Park Planning and Construction. Geography and Geographic Information Science, 25, 89-93+98.

[9] Niu, Q. (2017) GIS Technology Application Guide for Urban and Rural Planning, GIS Method and Classic Analysis. China Construction Industry Press, Beijing, 164.

[10] Zhu, L.J., Liu, X.J. and Ren, J. (2013) The Bearing Capacity of Park Green Space in Zhengzhou City and the Safety Evaluation of Disaster Prevention Park. Fujian Forestry Science and Technology, 40, 147-151.

[11] Sun, Y.N. (2019) Building a Resilient City to Enhance the City's Ability to Resist Risks. China Emergency Management, No. 5, 14-15. 